\title{
Construction of capacities from overlap indexes
}

\author{
José Antonio Sanz ${ }^{1}$, Mikel Galar ${ }^{1}$ Radko Mesiar ${ }^{23}$ Anna Kolesárová ${ }^{4}$, \\ Humberto Bustince ${ }^{1}$, Javier Fernandez ${ }^{1}$, and Javier Montero ${ }^{5}$ \\ 1 Departamento of Automática y Computación and the Institute of Smart Cities, Universidad \\ Publica de Navarra, Navarra, 31006 Spain \\ 2 Slovak University of Technology, Radlinskeho 11, Bratislava, Slovakia \\ 3 Institute of Information Theory and Automation, Academy of Sciences of the Czech \\ Republic, 18208 Prague, Czech Republic \\ 4 Institute of Information Engineering, Automation and Mathematics, Slovak University of \\ Technology, 81005 Bratislava, Slovakia \\ 5 Department of Statistics and Operations Research I, Faculty of Mathematics, Universidad \\ Complutense de Madrid, Plaza de Ciencias 3, 28040 Madrid, Spain.
}

\begin{abstract}
In this chapter, we show how the concepts of overlap function and overlap index can be used to define fuzzy measures which depend on the specific data of each considered problem.
\end{abstract}

Keywords: Overlap function, Capacity, Fuzzy measure.

\section{Introduction}

In many problems, it is crucial to find a relation between groups of data. Such relation can be expressed, for instance, in terms of an appropriate fuzzy measure or capacity([10, 21]) which reflects the way the different data are connected to each other [20].

In this chapter, taking into account this fact and following the developments in [8], we introduce a method to build capacities $([20,21])$ directly from the data (inputs) of a given problem. In order to do so, we make use of the notions of overlap function and overlap index $([5,12,13,7,4,14,16])$ for constructing capacities which reflect how different data are related to each other.

This paper is organized as follows: after providing some preliminaries, we analyse, in Section 3, some properties of overlap functions and indexes. In Sections 4 we discuss a method for constructing capacities from overlap functions and overlap indexes. Finally, we present some conclusions and references.

\section{Preliminaries}

Given a referential set (or universe) $U$, a fuzzy set $A$ over $U$ is defined in terms of a mapping $\mu_{A}$ :

$$
\mu_{A}: U \rightarrow[0,1]
$$


For simplicity, we write $A(i)$ instead of $\mu_{A}(i)$. We denote by $F S(U)$ the space of all fuzzy sets defined over $U$. We will only deal with fuzzy sets defined over a finite referential set $U$, so we can consider $U=\{1, \ldots, n\}$. $\operatorname{card}(U)$ stands for the cardinality of $U$.

We consider in $F S(U)$ the usual partial order defined by Zadeh. Union and intersection between fuzzy sets are defined by means of the max and min, respectively. We say that $\left(x_{1}, \ldots, x_{n}\right) \leq\left(y_{1}, \ldots, y_{n}\right)$ if and only if $x_{i} \leq y_{i}$ for every $i \in U$.

By abuse of notation, we denote by $\emptyset$ empty set (that is, the fuzzy set where the membership values of all the elements are equal to 0 ), and by $U$ the fuzzy set with all its memberships equal to 1 .

The support of a fuzzy set $A \in F S(U)$ is given by:

$$
\operatorname{supp}(A)=\{i \in U \mid A(i) \neq 0\} .
$$

We say that $A$ is a full fuzzy set if $\operatorname{supp}(A)=U$. To distinguish fuzzy sets from the classical subsets of $U$, we will use the notation $\widetilde{A}$ for the latter.

Let $\widetilde{A} \subseteq U$ and $t \in[0,1]$. By $t \widetilde{A}$ we denote the fuzzy set given by:

$$
t \widetilde{A}(i)= \begin{cases}t & \text { if } i \in \widetilde{A} \\ 0 & \text { otherwise }\end{cases}
$$

By abuse of notation, we write $1_{\widetilde{A}}$ to denote $1 \widetilde{A}$ for every $\widetilde{A} \subseteq U$, since $1 \widetilde{A}$ equals the characteristic function of the set $\widetilde{A}$. Note that this definition corresponds to the basic function $b(\widetilde{A}, t)$ introduced by Benvenuti et al. in 2002 ([2]).

Given a function $F:[0,1]^{k} \rightarrow[0,1]$ (with $k \in \mathbb{N}$ ) and $k$ fuzzy sets $A_{k} \in F S(U)$, the symbol $F\left(A_{1}, \ldots, A_{k}\right)$ denotes the fuzzy set over $U$ whose membership function is given by:

$$
F\left(A_{1}, \ldots, A_{k}\right)(i)=F\left(A_{1}(i), \ldots, A_{k}(i)\right) .
$$

Definition 1. Let $n \geq 2$. An n-dimensional aggregation function $([6,1,11,9,15,17$, 18]) is a mapping $M:[0,1]^{n} \rightarrow[0,1]$ such that:

1. $M(0, \cdots, 0)=0$ and $M(1, \ldots, 1)=1$;

2. $M$ is increasing.

\subsection{Capacities}

In the following, we recall some basic notions concerning capacities [21].

Definition 2. Let $U=\{1,2, \ldots, n\}$. A capacity (or non-additive measure) over $U$ is a mapping $m: 2^{U} \rightarrow[0,1]$ such that

1. $m(\emptyset)=0$ and $m(U)=1$;

2. If $\widetilde{A} \subset \widetilde{B}$ then $m(\widetilde{A}) \leq m(\widetilde{B})$.

Example 1. 1. Any probability measure yields an example of a capacity. 
2. The bottom capacity is defined by

$$
m_{*}(\widetilde{A})= \begin{cases}1 & \text { if } \widetilde{A}=U ; \\ 0 & \text { otherwise }\end{cases}
$$

3. The top capacity is defined by

$$
m^{*}(\widetilde{A})= \begin{cases}0 & \text { if } \widetilde{A}=\emptyset \\ 1 & \text { otherwise }\end{cases}
$$

Definition 3. If $m$ is a capacity over $U=\{1, \ldots, n\}$, then:

1. $m$ is called additive if $m(\widetilde{A} \cup \widetilde{B})=m(\widetilde{A})+m(\widetilde{B})$ whenever $\widetilde{A} \cap \widetilde{B}=\emptyset$.

2. $m$ is called symmetric if $m(\widetilde{A})=m(\widetilde{B})$ whenever $\operatorname{card}(\widetilde{A})=\operatorname{card}(\widetilde{B})$.

3. $m$ is called supermodular (submodular) if $m(\widetilde{A} \cup \widetilde{B})+m(\widetilde{A} \cap \widetilde{B}) \geq m(\widetilde{A})+m(\widetilde{B})$ $(m(\widetilde{A} \cup \widetilde{B})+m(\widetilde{A} \cap \widetilde{B}) \leq m(\widetilde{A})+m(\widetilde{B}))$ for every $\widetilde{A}, \widetilde{B} \in 2^{U}$.

4. $m$ is called modular if it is supermodular and submodular.

Remark 1 Since we have $m(\emptyset)=0$ for every capacity $m$, additivity and modularity are equivalent properties of capacities.

Capacities can be obtained from aggregation functions as follows.

Proposition 1. $[3,21]$ Let $m: 2^{U} \rightarrow[0,1]$ be a set function. The following items are equivalent.

1. $m$ is a capacity.

2. There exists an aggregation function $M:[0,1]^{n} \rightarrow[0,1]$ such that, for every $\widetilde{A} \in 2^{U}$

$$
m(\widetilde{A})=M\left(1_{\widetilde{A}}\right) .
$$

\section{Overlap functions and overlap indexes}

The concept of overlap function was extensively studied in [7]. Here we make a revision of the most relevant definitions and results for the present work.

Definition 4. An overlap function is a mapping $G_{O}:[0,1]^{2} \rightarrow[0,1]$ such that:

1. $G_{O}(x, y)=G_{O}(y, x)$ for every $x, y \in[0,1]$;

2. $G_{O}(x, y)=0$ if and only if $x y=0$;

3. $G_{O}(x, y)=1$ if and only if $x y=1$;

4. $G_{O}$ is increasing;

5. $G_{O}$ is continuous.

Overlap function can be seen as a generalization of continuous t-norms without divisors of zero. The class of all overlap functions is convex.

Overlap functions can be used to build overlap indexes by aggregating them. We start by recalling some basic notions about the idea of an overlap index and we will formalize the construction method in Theorem 1. 
Definition 5. An overlap index is a mapping $O: F S(U) \times F S(U) \rightarrow[0,1]$ such that

(O1) $O(A, B)=0$ if and only if $A$ and $B$ have disjoint supports; that is, $A(i) B(i)=0$ for every $i \in U$;

(O3) $O(A, B)=O(B, A)$;

(O4) If $B \subseteq C$, then $O(A, B) \leq O(A, C)$.

An overlap index such that

(O2') $O(A, B)=1$ if there exists $i \in U$ such that $A(i)=B(i)=1$

is called a normal overlap index.

Remark 2 In the original definition of overlap index [12], condition (O2) states that

$$
O(A, B)=1 \text { if } A(i)=0 \text { or } B(i)=1 \text { or } A(i) B(i)=0
$$

for all $i \in U$. For $A=\emptyset$ we obtain the following contradiction: (O1) implies that $O(A, A)=0$ wheereas $(O 2)$ implies $O(A, A)=1$. Therefore we removed condition $(O 2)$ from the definition of an overlap index.

Example 2. 1. The first example of overlap index in the literature is Zadeh's consistency index ([22]):

$$
O_{Z}(A, B)=\max _{i=1}^{n}(\min (A(i), B(i))) .
$$

Note that $O_{Z}$ is normal.

2. Let $M:[0,1]^{2} \rightarrow[0,1]$ be a symmetric aggregation function such that $M(x, y)=$ 0 if and only if $x y=0$. We have that

$$
O_{M, Z}(A, B)=\max _{i=1}^{n}(M(A(i), B(i)))
$$

is a normal overlap index that generalizes Zadeh's index.

Remark 3 For each overlap index $O: F S(U) \times F S(U) \rightarrow[0,1]$, the function $M_{O}$ : $[0,1]^{n} \rightarrow[0,1]$ given by

$$
M_{O}(E)=\frac{O(E, U)}{O(U, U)}
$$

with $E \in[0,1]^{n}$, is an aggregation function without divisors of zero.

\subsection{Modularity of overlap indexes}

We are going to analyze several properties of overlap indexes that will be relevant for the construction of capacities from them. We first introduce the idea of symmetry for overlap indexes.

Definition 6. Let $O: F S(U) \times F S(U) \rightarrow[0,1]$ be an overlap index and let $E \in$ $F S(U) . O$ is E-symmetric if for every $A, B \in F S(U)$ such that $\operatorname{card}(\operatorname{supp}(A))=$ $\operatorname{card}(\operatorname{supp}(B))$ it holds that:

$$
O(A, E)=O(B, E) .
$$


Example 3. 1. Every overlap index $O$ is $E$-symmetric if $E=\emptyset \in F S(U)$.

2. Consider the strongest overlap index:

$$
O_{s}(A, B)= \begin{cases}0 & \text { if } A, B \text { are disjoint fuzzy sets } \\ 1 & \text { otherwise }\end{cases}
$$

We have that $O_{s}$ is $E$-symmetric for every full set $E$.

Note that an overlap index can not be $E$-symmetric unless $E$ is a full fuzzy set, as the next result shows.

Proposition 2. If $O$ is an overlap index which is E-symmetric with respect to some fuzzy set $E \in F S(U), E \neq \emptyset$, then $E$ is a full fuzzy set.

Proof. Assume that $E$ is not a full fuzzy set and that

$$
k=\min (\operatorname{card}(\operatorname{supp}(E)), n-\operatorname{card}(\operatorname{supp}(E)))>0 .
$$

Let $\widetilde{A} \subseteq \operatorname{supp}(E)$ and $\widetilde{B} \subseteq U \backslash \operatorname{supp}(E)$ with $\operatorname{card}(\widetilde{A})=\operatorname{card}(\widetilde{B})=k$. Consider the fuzzy sets

$$
A=1_{\widetilde{A}} \text { and } B=1_{\widetilde{B}} .
$$

We have that $O(E, A)>0$ (since $E$ and $A$ are not mutually disjoint) whereas $O(E, B)=$ 0 . Therefore, $O$ can not be $E$-symmetric.

Now we consider the notion of modularity.

Definition 7. Let $O: F S(U) \times F S(U) \rightarrow[0,1]$ be an overlap index and let $E \in$ $F S(U)$.

1. $O$ is called E-supermodular if $O(E, A \cap B)+O(E, A \cup B) \geq O(E, A)+O(E, B)$ holds for all $A, B \in F S(U)$. Similarly, $O$ is called $E$-submodular if $O(E, A \cap B)+$ $O(E, A \cup B) \leq O(E, A)+O(E, B)$ for all $A, B \in F S(U)$.

2. If $O$ is E-submodular and E-supermodular, then $O$ is simply called E-modular.

Example 4. 1. Every overlap index $O$ is $E$-modular for $E=\emptyset \in F S(U)$.

2. The overlap index $O_{\pi}$ is $E$-modular for every fuzzy set $E$.

3. $O_{Z}$ is $E$-submodular but not $E$-modular.

The following construction method of overlap indexes by means of aggregation functions can be found in [13].

Theorem 1. Let $M:[0,1]^{n} \rightarrow[0,1]$ be an aggregation function such that $M\left(x_{1}, \ldots, x_{n}\right)=$ 0 if and only if $x_{1}=\cdots=x_{n}=0$ and let $G_{O}:[0,1]^{2} \rightarrow[0,1]$ be an overlap function. The mapping $O: F S(U) \times F S(U) \rightarrow[0,1]$ given by

$$
O(A, B)=M\left(G_{O}(A(1), B(1)), \ldots, G_{O}(A(n), B(n))\right)
$$

is a normal overlap index in the sense of Definition 5.

Conversely, if $G_{O}$ is an overlap function and $M:[0,1]^{n} \rightarrow[0,1]$ is an aggregation function such that $O$ defined by Equation 1 is an overlap index, then $M\left(x_{1}, \ldots, x_{n}\right)=$ 0 if and only if $x_{1}=\cdots=x_{n}=0$. 


\section{Capacities from Overlap Indexes, and Overlap Functions}

In this section, we present the core of the present chapter. Taking into account the usual construction of Bayesian conditional probabilities, we follow an analogous procedure to build a capacity from an overlap function.

We start introducing a bit of notation. Let $E \in F S(U)$ be a fixed non-empty fuzzy set (that is, with at least one membership different from zero). Given $\widetilde{A} \in 2^{U}$, let us define a fuzzy set $E_{\widetilde{A}}$ induced by $E$ as follows:

$$
E_{\widetilde{A}}(i)= \begin{cases}E(i) & \text { if } i \in \widetilde{A} \\ 0 & \text { otherwise }\end{cases}
$$

Observe that $E_{\widetilde{A}}$ is the fuzzy intersection of the fuzzy set $E$ and the crisp set $\widetilde{A}$, since

$$
E_{\widetilde{A}}(i)=\min \left(1_{\widetilde{A}}(i), E(i)\right)
$$

Therefore, any aggregation function with no zero divisors could also be used instead of the minimum in this definition for the subsequent developments.

Now we are ready to introduce our main result.

Theorem 2. If $E \in F S(U)$ is a fixed, non-empty fuzzy set, then the mapping $m_{O, E}$ : $2^{U} \rightarrow[0,1]$ given by

$$
m_{O, E}(\widetilde{A})=\frac{1}{O(E, E)} O\left(E, E_{\widetilde{A}}\right)
$$

is a capacity for every overlap index $O$.

Proof. First of all observe that $m_{O, E}$ is well defined since $O(E, E) \neq 0$ and $O\left(E, E_{\widetilde{A}}\right) \leq$ $O(E, E)$. If $\widetilde{A}=U$, then it follows that $E_{\widetilde{A}}=E$, so we have that $m_{O, E}(\widetilde{A})=1$. Moreover, if $\widetilde{A}=\emptyset$, then $E_{\widetilde{A}}(i)=0$ for every $i \in U$. So, in particular, $O\left(E_{\widetilde{A}}, E\right)=0$. Finally, if $\widetilde{A} \subset \widetilde{B}$, then it follows that $E_{\widetilde{A}} \subseteq E_{\widetilde{B}}$, so, in particular, $O\left(E, E_{\widetilde{A}}\right) \leq$ $O\left(E, E_{\widetilde{B}}\right)$ and hence $m_{O, E}(\widetilde{A}) \leq m_{O, E}(\widetilde{B})$.

Recall that Benvenuti et al. ([3]) defined for each aggregation function $M:[0,1]^{n} \rightarrow$ $[0,1]$ and $e \in] 0,1]$ such that $M(E)>0$, where $E=(e, \ldots, e)$, a capacity $m_{M, e}$ : $2^{U} \rightarrow[0,1]$ given by

$$
m_{M, e}(\widetilde{A})=\frac{M(e \widetilde{A})}{M(E)}
$$

(for $e=1$ see also Proposition 1). Obviously, in the terms of Theorem 2, $m_{O, E}=$ $m_{M_{O}, e}$. Here $M_{O}$ was defined in Remark 3.

Remark 4 Observe that, for a fixed full probability measure $P$ on $U$, if we consider the overlap index $O_{P}$ introduced in Remark 3, we recover the definition of Bayesian conditional probabilities, i.e., $m_{O_{P}, E}=P_{\text {suppE }}$.

Of course, the question of how two of these measures relate to each other arises. 
Proposition 3. Let $O$ be an overlap index. For all full fuzzy sets $E_{1}, E_{2} \in F S(U)$, the following statements are equivalent:

1. $m_{O, E_{1}}(\widetilde{A}) \leq m_{O, E_{2}}(\widetilde{A})$ for every $\widetilde{A} \in 2^{U}$.

2. $\min _{\widetilde{A} \in 2^{U}} \frac{O\left(E_{2}, \widetilde{A}_{E_{2}}\right)}{O\left(E_{1}, \widetilde{A}_{E_{1}}\right)}=\frac{O\left(E_{2}, E_{2}\right)}{O\left(E_{1}, E_{1}\right)}$

Proof. The inequality $m_{O, E_{1}}(\widetilde{A}) \leq m_{O, E_{2}}(\widetilde{A})$ implies that

$$
\frac{O\left(E_{2}, E_{2}\right)}{O\left(E_{1}, E_{1}\right)} \leq \frac{O\left(E_{2}, \widetilde{A}_{E_{2}}\right)}{O\left(E_{1}, \widetilde{A}_{E_{1}}\right)}
$$

for every $\widetilde{A} \in 2^{U}$, so (2) holds. Conversely, if (2) holds, then Equation 2 is satisfied as well and we obtain (1).

Corollary 1. Let $E_{1}, E_{2} \in F S(U)$ such that $O\left(E_{1}, E_{1}\right)=1$. If $E_{1} \subseteq E_{2}$, then $m_{O, E_{1}}(\widetilde{A}) \leq m_{O, E_{2}}(\widetilde{A})$ for every $\widetilde{A} \in 2^{U}$.

Proof. If $E_{1} \subseteq E_{2}$, then we have that $1 \geq O\left(E_{2}, E_{2}\right) \geq O\left(E_{1}, E_{1}\right)=1$. Consequently,

$$
1=\frac{O\left(E_{2}, E_{2}\right)}{O\left(E_{1}, E_{1}\right)} \leq \frac{O\left(E_{2}, \widetilde{A}_{E_{2}}\right)}{O\left(E_{1}, \widetilde{A}_{E_{1}}\right)}
$$

for every $\widetilde{A} \in 2^{U}$, which implies that $m_{O, E_{1}}(\widetilde{A}) \leq m_{O, E_{2}}(\widetilde{A})$ for every $\widetilde{A} \in 2^{U}$.

In order to reverse the construction method and get an overlap function from a measure, we need the concept of contraction.

Definition 8. Let $E \in F S(U)$. The contraction to $E$ (or E-contraction) is the mapping $C_{E}: F S(U) \rightarrow F S(U)$ defined by:

$$
C_{E}(A)=\{(i, E(i) A(i)) \mid i \in U\} .
$$

Remark 5 The definition of contraction can be generalized by substituting the product with any other t-norm or even an overlap function. We postpone the analysis of the resulting operators to future works.

Let us continue by introducing some notations. For a fixed fuzzy set $E \in F S(U)$ and for $\widetilde{A} \in 2^{U}$, we define

$$
C l(E, \widetilde{A})=\left\{A \in F S(U) \mid A \subseteq E_{\widetilde{A}} \text { and } A \nsubseteq E_{\widetilde{B}} \text { for every } \widetilde{B} \subset \widetilde{A}\right\} .
$$

The proof of the following lemma is straightforward.

Lemma 1. Let $E \in F S(U)$. Then $C l(E, \widetilde{A})=\emptyset$ for every $\widetilde{A} \in 2^{U}$ such that $\widetilde{A} \cap$ $\operatorname{supp}(E)=\emptyset$.

Then we can state the following. 
Proposition 4. Let $E$ be a full fuzzy set. The family $(C l(E, \widetilde{A}))_{\widetilde{A} \in 2^{U}}$ is a partition of the set $\{A \in F S(U) \mid A \subseteq E\}$.

Proof. For any $A \subseteq E$ let's take $\widetilde{A}=\operatorname{supp}(A)$. Then, and only then $A \subseteq E_{\widetilde{A}}$ and for any $\widetilde{B}$ which is a proper subset of $\widetilde{A}, A \nsubseteq E_{\widetilde{B}}$, i.e., $A \in C l(E, \widetilde{A})$. The fact that $C l(E, \widetilde{A}) \cap C l(E, \widetilde{B})=\emptyset$ for $\widetilde{A} \neq \widetilde{B}$ is trivial. Hence $\left\{C l(E, \widetilde{A}) \mid \widetilde{A} \in 2^{U}\right\}$ is a partition of $\{A \in F S(U) \mid A \subseteq E\}$.

Now we can show how to recover overlap indexes from capacities.

Theorem 3. Let $m$ be a capacity such that $m(\widetilde{A})=0$ if and only if $\widetilde{A}=\emptyset$. If $E$ is a full fuzzy set, then the function $O_{E, m}: F S(U) \times F S(U) \rightarrow[0,1]$ defined by:

$$
O_{E, m}(A, B)= \begin{cases}m(\widetilde{A}) & \text { if } A \cap B \in C l(E, \widetilde{A}) ; \\ 1 & \text { otherwise }\end{cases}
$$

is a normal overlap index such that the capacity induced by $O_{E, m}$ is equal to $\mathrm{m}$.

Proof. First of all, due to Proposition 4, $O_{E, m}$ is well defined. Let us prove that $O_{E, m}$ is an overlap index.

(O1) Assume that $O_{E, m}(A, B)=0$. Since $m(\widetilde{A}) \neq 0$ for every $\widetilde{A} \neq \emptyset$, this happens if and only if $A \cap B \in C l(E, \emptyset)$, i.e., if and only if $A$ and $B$ have disjoint supports.

(O3) Symmetry is obvious from the definition.

(O4) Let $A \in F S(U)$ be arbitrary, but fixed and let $B \subseteq C$. If $A \cap C \nsubseteq E$, then $O_{E, m}(A, C)=1 \geq O_{E, m}(A, B)$. Now let us assume that $A \cap C \subseteq E$. From Proposition 4 and the fact that $A \cap B \subseteq A \cap C$, it follows that there exist $\widetilde{A}, \widetilde{B} \in 2^{U}$ with $\widetilde{B} \subseteq \widetilde{A}$ such that $A \cap C \in C l(E, \widetilde{A})$ and $A \cap B \in C l(E, \widetilde{B})$. Since $m$ is a capacity, we have that $m(\widetilde{A}) \leq m(\widetilde{B})$ and therefore $O_{E, m}(A, B) \leq O_{E, m}(A, C)$.

(O2') Note that $U=U \cap U$. So if $E \neq U$ it follows that $O_{E, m}(U, U)=1$, whereas if $E=U$, we have that $U \in C l(U, U)$ and $O_{E, m}(U, U)=m(U)=1$.

Finally, note that $E_{\widetilde{A}}=E_{\widetilde{A}} \cap E \in C l(E, \widetilde{A})$ for every $\widetilde{A} \in 2^{U}$, which concludes the proof of the theorem since

$$
m_{O_{E, m}, E}(\widetilde{A})=\frac{1}{O_{E, m}(E, E)} O_{E, m}\left(E, E_{\widetilde{A}}\right)=m(\widetilde{A}) .
$$

This completes the proof.

Theorem 3 can be extended to include non-strict measures as follows.

Corollary 2. Consider a capacity $m$. Let $\widetilde{A}_{0}=\{i \in U \mid m(\{i\})=0\}$. Suppose that $E$ is a fuzzy set such that $E(i) \neq 0$ for $i \notin \widetilde{A}_{0}$. The function $O: F S(U) \times F S(U) \rightarrow[0,1]$ given by

$$
O_{E, m, \widetilde{A}_{0}}(A, B)= \begin{cases}m(\widetilde{A}) & \text { if } A \cap B \in C l\left(E, \widetilde{A} \backslash \widetilde{A}_{0}\right) ; \\ 1 & \text { otherwise }\end{cases}
$$

is an overlap index. 
Proof. Symmetry and monotonicity are clear. We only need to check that (O1) holds. To see (O1), note that $O(A, B)=0$ if and only if $A \cap B \in C l\left(E, \widetilde{A} \backslash \widetilde{A}_{0}\right)$ for some $\widetilde{A} \in 2^{U}$ such that $m(\widetilde{A})=0$. But, $m(\widetilde{A})=0$ if and only if $\widetilde{A} \subseteq \widetilde{A}_{0}$, due to the monotonicity of capacities. So, $\min (A(i), B(i))=0$ if $i \in \widetilde{A}_{0}$

Example 5. For the bottom capacity $m_{*}$, we obtain $\widetilde{A}_{0}=\left\{i \in U \mid m_{*}(\{i\})=0\right\}=$ $U$. Thus, setting $E=\emptyset$ yields the following overlap function:

$$
O_{E, m_{*}, U}(A, B)= \begin{cases}0 & \text { if } A \cap B=\emptyset ; \\ 1 & \text { otherwise }\end{cases}
$$

which is the strongest overlap index.

Corollary 3. For every capacity $m$ there exists a fuzzy set $E$ and a continuous overlap index $O_{E, m}$ such that the measure induced by $O_{E, m}$ is equal to $m$.

Proof. It is just a matter of using the overlap index defined by means of Theorem 3 and Corollary 2

\subsection{Construction of Capacities from Overlap Indexes Based on Overlap Functions}

We can make use of Theorem 1 to get capacities from overlap functions and aggregation functions.

Proposition 5. Let $M:[0,1]^{n} \rightarrow[0,1]$ be an aggregation function such that $M\left(x_{1}, \ldots, x_{n}\right)=$ 0 if and only if $x_{1}=\cdots=x_{n}=0$, let $G_{O}:[0,1]^{2} \rightarrow[0,1]$ be an overlap function and let $E \in F S(U)$ be a non-empty fuzzy set. The mapping $m_{E, M, G_{O}}: 2^{U} \rightarrow[0,1]$ is given by

$$
m_{E, M, G_{O}}(\widetilde{A})=\frac{1}{M\left(G_{O}(E)\right)} M\left(G_{O}\left(E(1), E_{\widetilde{A}}(1)\right), \ldots, G_{O}\left(E(n), E_{\widetilde{A}}(n)\right)\right),
$$

where $M\left(G_{O}(E)\right)=M\left(G_{O}(E(1), E(1)), \ldots, G_{O}(E(n), E(n))\right.$ is a capacity.

Note that if we take $E=U$, then we have $m_{U, M, G_{O}}(\widetilde{A})=M\left(1_{\widetilde{A}}\right)$.

Proposition 6. Let $M$ be an aggregation function as in Proposition 5. For any nonempty fuzzy set $E$, we have:

1. $m_{E, M, G_{O}}(\widetilde{A})=0$ if and only if $E(i)=0$ for every $i \in \widetilde{A}$;

2. $m_{E, M, G_{O}}(\widetilde{A})=1$ whenever $E(i) \neq 0$ for every $i \in \widetilde{A}$.

Proof. 
1. If $m_{E, M, G_{O}}(\widetilde{A})=0$, then

$$
\frac{1}{M\left(G_{O}(E)\right)} M\left(G_{O}\left(E(1), E_{\widetilde{A}}(1)\right), \ldots, G_{O}\left(E(n), E_{\widetilde{A}}(n)\right)\right)=0 .
$$

Since $M$ is an aggregation function, this implies that $G_{O}\left(E(i), E_{\widetilde{A}}(i)\right)=0$ for every $i=1, \ldots, n$. From the definition of an overlap function, this happens only if $E(i) E_{\widetilde{A}}(i)=0$ for every $i=1, \ldots, n$. If $E_{\widetilde{A}}(i) \neq 0$ it follows that $E(i)=0$, which is impossible due to the definition of $E_{\widetilde{A}}$. Therefore, we infer that $E_{\widetilde{A}}(i)=0$ for every $i \in \widetilde{A}$, that is, if $i \in \widetilde{A}$ then $E(i)=0$. The other direction follows from the fact that $E(i)=0$ for every $i \in \widetilde{A}$ implies that $E_{\widetilde{A}}(i)=0$ for every $i \in U$.

2. If $\widetilde{A}$ is as in the statement of this property, then we obtain $E_{\widetilde{A}}=E$, and the result follows from the monotonicity of aggregation and overlap functions.

The following corollary is a straightforward consequence of the previous result.

Corollary 4. Let $M$ be an aggregation function as in Proposition 5. For any non-empty fuzzy set $E$, we have:

1. $m_{E, M, G_{O}}$ satisfies the property

$$
m_{E, M, G_{O}}(\widetilde{A})=0 \text { if and only if } \widetilde{A}=\emptyset
$$

if and only if $E$ is a full fuzzy set.

2. $m_{E, M, G_{O}}(\operatorname{supp}(E))=1$.

Theorem 4. For a fixed overlap function $G_{O}$ and an n-ary aggregation function $M$ as in Proposition 5, the following claims are equivalent:

1. For each non-empty fuzzy subset $E \in F S(U)$, the measure $m_{E, M, G_{O}}$ is additive.

2. $M$ is modular, i.e., $M(\max (x, y))+M(\min (x, y))=M(x)+M(y)$ for all $x, y \in$ $[0,1]^{n}$.

Proof. Observe first that any modular aggregation function $M$ such that $M\left(x_{1}, . ., x_{n}\right)=$ 0 only if $x_{1}=\cdots=x_{n}=0$ has the form $M\left(x_{1}, \ldots, x_{n}\right)=\sum_{i=1}^{n} f_{i}\left(x_{i}\right)$, where each $f_{i}:[0,1] \rightarrow[0,1]$ is increasing, $f_{i}(x)=0$ only if $x=0$, and $\sum f_{i}(1)=1$, see, e.g., [19]. To see the necessity, observe that the additivity of $m_{E, M, G_{O}}$ implies that

$$
\begin{gathered}
M\left(G_{O}\left(E(1), E_{\widetilde{A}}(1)\right), \ldots, G_{O}\left(E(n), E_{\widetilde{A}}(n)\right)\right)= \\
M\left(G_{O}\left(\left(E(1), E_{\widetilde{A}}(1)\right), 0, \ldots, 0\right)\right)+M\left(0, \ldots, 0, G_{O}\left(E(n), E_{\widetilde{A}}(n)\right)\right) .
\end{gathered}
$$

In view of the mean value theorem for overlap functions, this equality is equivalent to

$$
M\left(x_{1}, \ldots, x_{n}\right)=M\left(x_{1}, 0, \ldots, 0\right)+M\left(0, x_{2}, 0, \ldots, 0\right)+\cdots+M\left(0, \ldots, 0, x_{n}\right)
$$

for every $x_{1}, \ldots, x_{n} \in[0,1]$. Defining $f_{i}(x)=M(0,0, \ldots, 0, x, 0, \ldots, 0)$, where the $x$ is in the $i$-th position, the result follows. To see the converse, observe that $M\left(x_{1}, \ldots, x_{n}\right)=$ $\sum_{i=1}^{n} f_{i}\left(x_{i}\right)$ is an aggregation function such that $M\left(x_{1}, \ldots, x_{n}\right)=0$ if and only if $x_{1}=\cdots=x_{n}=0$.

Observe that if $M$ satisfies the requirements of the previous theorem, then $m_{E, M, G_{O}}$ is additive for all $G_{O}$ and all $E \neq \emptyset$. 


\section{Conclusions}

In this chapter we have made a summary of the developments in [8] and discussed a method to build capacities from overlap indexes and overlap measures. In this way, relationships among data in the problem can be taken into account in order to build an appropriate measure which will later be used to determine the aggregation procedure.

In future research, we intend to analyse aggregations that make use of our measures, such as Sugeno, Shilkret, Choquet or copula-based integrals, and compare the obtained results using other aggregations in different problems, such as, for instance, digital fingerprint recognition or decision making.

\section{Acknowledgments}

The work has been supported by projects TIN2013-40765-P and TIN2015-66471-P of the Spanish Ministry of Science, by grant VEGA 1/0420/15 and by grant VEGA $1 / 0419 / 13$.

\section{References}

[1] A. Amo, J. Montero, E. Molina, Representation of consistent recursive rules. European Journal of Operational Research, 130, 29-53, 2001.

[2] P. Benvenuti, R. Mesiar, Pseudo-additive measures and triangular-norm-based conditioning. Annals of Mathematics and Artificial Intelligence, 35, 63-69, 2002.

[3] P. Benvenuti, D. Vivona, M. Divari, Aggregation operators and associated fuzzy measures. International Journal of Uncertainty, Fuzziness and Knowledge-Based Systems, 9, 197204, 2001.

[4] H. Bustince, M. Pagola, R. Mesiar, E. Hüllermeier, F. Herrera, Grouping, Overlap, and Generalized Bientropic Functions for Fuzzy Modeling of Pairwise Comparisons. IEEE Transactions on Fuzzy Systems, 20(3), 405-415, 2012.

[5] H. Bustince, E. Barrenechea, M. Pagola, F. Soria, Weak fuzzy S-subsethood measures: Overlap index. International Journal of Uncertainty Fuzziness and Knowledge-Based Systems, 14(5), 537-560, 2006.

[6] H. Bustince, J. Montero, E. Barrenechea, M. Pagola, Semiautoduality in a restricted family of aggregation operators. Fuzzy Sets and Systems, 158, 1360-1377, 2007.

[7] H. Bustince, J. Fernandez, R. Mesiar, J. Montero, R. Orduna, Overlap functions. Nonlinear Analysis: Theory, Methods \& Applications, 72(3-4), 1488-1499, 2010.

[8] H. Bustince, D. Paternain, M. Pagola, P. Sussner, A. Kolesárová, R. Mesiar, Capacities and Overlap Indexes with an Application in Fuzzy Rule- Based Classification Systems, Fuzzy Sets and Systems, accepted for publication, in press.

[9] T. Calvo, A. Kolesárová, M. Komorníková and R. Mesiar: Aggregation Operators: Properties, Classes and Construction Methods. In T. Calvo, G. Mayor and R. Mesiar (Eds.): Aggregation Operators. New Trends and Applications. Physica-Verlag, Heidelberg, pp. 3104, 2002.

[10] G. Choquet, Theory of capacities. Ann. Inst. Fourier 5, 131-295, 1953-54.

[11] V. Cutello, J. Montero, Recursive connective rules. International Journal of Intelligent Systems, 14, 3-20, 1999.

[12] D. Dubois, W. Ostasiewicz, H. Prade, Fuzzy Sets: History and Basic Notions. In D. Dubois, H. Prade (Eds.), Fundamentals of Fuzzy Sets. Kluwer, Boston, MA, pp. 21-124, 2000. 
[13] S. Garcia, H. Bustince, E. Hüllermeier, R. Mesiar, N. Pal, A. Pradera, Overlap Indices: Construction of and Application to Interpolative Fuzzy Systems, IEEE Transactions on Fuzzy Systems 23, 1259-1273, 2015.

[14] D. Gomez, J.T. Rodriguez, J. Montero, H. Bustince, E. Barrenechea, N-dimensional overlap functions, Fuzzy Sets and Systems 287, 57-75, 2016.

[15] M. Grabisch, J.-L. Marichal, R. Mesiar, E. Pap, Aggregation Functions. Cambridge University Press, Cambridge, 2009.

[16] A. Jurio, H. Bustince, M. Pagola, A. Pradera, R.R. Yager, Some properties of overlap and grouping functions and their application to image thresholding. Fuzzy Sets and Systems, 229, 69-90, 2013.

[17] D. Gómez, J. Montero, A discussion on aggregation operators. Kybernetika 40, 107-120, 2004.

[18] G.J. Klir, T.A. Folger, Fuzzy Sets, Uncertainty and Information. Prentice Hall, Englewood Cliffs, NJ, 1988.

[19] R. Mesiar, A. Mesiarová-Zemánková, The Ordered Modular Averages. IEEE Transactions on Fuzzy Systems, 19, 42-50, 2011.

[20] E. Pap, Handbook of Measure Theory, Part I, PartII. Elsevier, Amsterdam, 2002.

[21] V. Torra, Y. Narukawa, M. Sugeno, Eds., Non-Additive Measures, Theory and Applications. Studies in Fuzziness and Soft Computing, 310, 2014.

[22] L.A. Zadeh, Fuzzy sets. Information Control, 8, 338-353, 1965. 